\title{
ESTIMACIÓN DEL CONTENIDO Y CAPTURA POTENCIAL DE CARBONO EN LA BIOMASA ARBÓREA DE BOSQUES NATIVOS DEL ESPINAL (Entre Ríos, Argentina)
}

\author{
Ledesma, S. G. ${ }^{1} ;$ Sione, S. M. J. ${ }^{1}$; Oszust, J. D. ${ }^{1} ;$ Rosenberger, L. J. ${ }^{1}$
}

\begin{abstract}
RESUMEN
Los bosques nativos intervienen en el ciclo global del carbono (C), dado su rol de secuestradores de $\mathrm{CO}_{2}$. El objetivo de esta investigación fue estimar el stock y la tasa anual de captura de $\mathrm{C}$ en bosques del Espinal entrerriano. Se evaluaron seis bosques semixerofíticos, estimándose biomasa arbórea aérea, stock de $\mathrm{C}$ y tasa de captura de $\mathrm{C}$ mediante modelos alométricos locales y tasas de crecimiento por especie. El contenido de biomasa osciló entre 52,13 y 111,82 t MS/ha; en promedio 92,68 \pm 23,13 t MS/ha. El stock de C promedió 43,99 $\pm 10,43$ t/ha; variando entre 26,02 t/ha y 52,78 t/ha. Se observó una alta heterogeneidad entre bosques, acorde a su composición específica y estructura diamétrica. La tasa de captura de C osciló entre 0,53 y 0,95 t C/ha/año, equivalente a una captura de entre 1,94 y 3,49 t CO 2 /ha/año. Este estudio genera información de relevancia para el diseño de estrategias de manejo de bosques nativos que contribuyan a la mitigación del cambio climático global.
\end{abstract}

Palabras clave: bosques nativos, biomasa, stock de C, tasa de captura.

1.- Facultad de Ciencias Agropecuarias, Universidad Nacional de Entre Ríos. Ruta 11 - Km 10,5. 3101, Oro Verde, Entre Ríos, Argentina. Email: silvia.ledesma@fca.uner.edu.ar Manuscrito recibido el 14 de abril de 2020 y aceptado para su publicación el 5 de octubre de 2020.

Ledesma, S.G.; Sione, S.M.J.; Oszust, J.D.; Rosenberger, L.J. Estimación del contenido y captura potencial de carbono en la biomasa arbórea de bosques nativos del Espinal (Entre Ríos, Argentina).

FAVE - Ciencias Agrarias 20 (1): 331-345. CC BY-NC-SA 4.0 


\begin{abstract}
Content estimation and capture carbon potential in biomass tree of Espinal native forests (Entre Ríos, Argentine).

Native forests intervene in the global carbon cycle (C), given their role as $\mathrm{CO}_{2}$ sequestrators. The objective was to estimate the stock and the annual $\mathrm{C}$ capture rate in forests of the Espinal (Entre Ríos). Six forests were evaluated, estimating aerial tree biomass, $\mathrm{C}$ stock and $\mathrm{C}$ capture rate using local allometric models and growth rates by species. The biomass content ranged between 52.13 and $111.82 \mathrm{t} \mathrm{DM} / \mathrm{ha}$, on average $92.68 \pm 23.13 \mathrm{t} \mathrm{DM} / \mathrm{ha}$. The $\mathrm{C}$ stock averaged $43.99 \pm 10.43 \mathrm{t} / \mathrm{ha}$, varying between $26.02 \mathrm{t} / \mathrm{ha}$ and $52.78 \mathrm{t} / \mathrm{ha}$. It was observed a high heterogeneity between forests, according to their specific composition and diameter structure. The $\mathrm{C}$ capture rate ranged from 0.52 to $0.95 \mathrm{t} \mathrm{C/ha/year,} \mathrm{equivalent} \mathrm{to} \mathrm{a} \mathrm{capture} \mathrm{of} \mathrm{between} 1.94$ and 3,49 $\mathrm{t} \mathrm{CO}_{2} /$ ha/year. This study generates relevant information for the design of native forest management strategies that contribute to mitigate the global climate change.
\end{abstract}

Key words: native forest, biomass, $C$ stock, anual C capture rate.

\section{INTRODUCCION}

Las principales causas del aumento de la concentración de gases efecto invernadero (GEI) en la atmósfera están relacionadas con la actividad humana, fundamentalmente por el empleo de combustibles fósiles, cuya quema significa cerca de un $70 \%$ de las emisiones globales de dióxido de C $\left(\mathrm{CO}_{2}\right)$ (Ibárcena Escudero y Scheelje Bravo, 2003). La quema del material generado por la deforestación, sumado a la degradación de biomasa han sido señaladas también como importantes fuentes de emisiones de este gas, y es allí donde se vislumbran las principales posibilidades de mitigación (Barala y Guha, 2004; Canadell y Raupach, 2008; Martin y Thomas, 2011). Los bosques nativos y los sistemas agroforestales juegan un rol preponderante en el ciclo global del C, regulando el intercambio gaseoso entre la biomasa vegetal, el suelo y la atmósfera. En particular, el servicio ambiental que proveen estos ecosistemas como secuestradores de $\mathrm{CO}_{2}$ contribuye a mitigar el efecto invernadero (Yepes et al., 2010) resultando prioritario desarrollar estrategias de acción forestal tendientes a mitigar las emisiones de GEI (Barala y Guha, 2004; Janowiak et al., 2017; Ordóñez Díaz et al., 2015).

En Argentina, la Región del Espinal abarca una superficie total de $307.374 \mathrm{~km}^{2}$, según lo describe el Primer Inventario Nacional de Bosques Nativos (Secretaría de Ambiente y Desarrollo Sustentable de la Nación 2007) donde el bosque semixerofítico es el bioma dominante (Cabrera, 1976). El cambio de uso del suelo con destino a la producción agrícola y el manejo irracional del pastoreo del ganado han desencadenado procesos de degradación y eliminación de bosques nativos. Estudios recientes indican que, en esa región, la pérdida de bosques se ha dado a una tasa anual de 1,07\% (Sabattini et al., 2016). Esta si- 
tuación, similar a lo registrado en las otras áreas boscosas del país, llevó a la sanción de la Ley Nacional 26331 de Presupuestos Mínimos de protección de bosques nativos; en la que uno de los principales objetivos es el mantenimiento de estos ecosistemas por sus “...beneficios ambientales o por los daños ambientales que su ausencia genera$s e$ ". En ese contexto cobran cada vez más importancia los proyectos de reducción de emisiones por deforestación y degradación (REDD+) en las áreas de bosques nativos; ya que además de almacenar $\mathrm{C}$, ayudan a la protección hídrica, la conservación de la diversidad y brindan belleza escénica (Bishop y Landell-Mills, 2003).

La información que respalda la función de fijación de $\mathrm{C}$ realizada por los bosques es el cálculo de su biomasa, a partir de la cual es posible determinar el stock de C. En un estudio sobre la capacidad de fijación de C de bosques nativos, se destaca que América del Sur experimentó las mayores pérdidas netas de bosque en la primera década del siglo XXI (4 millones de hectáreas por año), lo cual le acarreó una pérdida total de cerca de 8,3 Pg C (1 Petagramo $=10^{12}$ kg) entre 1990 y 2015 (Segura y Madrigal, 2019). Si bien muchas estimaciones de este tipo se han realizado en base a la utilización de modelos matemáticos generales obtenidos en distintas partes del mundo, se ha reforzado últimamente la investigación que origina modelos locales de biomasa y de contenido de C; lo cual es importante para evitar sesgos en las estimaciones (Martin y Thomas, 2011; Sione et al., 2019 (1)). En tal sentido, se han desarrollado estudios para ajustar metodologías de estimación de biomasa y contenido de $\mathrm{C}$ para las especies arbóreas del Espinal y de otros bosques xerofíticos de Argentina (Bonino, 2006; Gaillard de Benitez et al., 2002; Sione et al.,
2019 (1) y (2)). Estos estudios aumentan la confiabilidad por cuanto generan, a nivel de ecosistemas locales, ecuaciones alométricas, valores de fracción de C de la biomasa y factores de expansión. En ese aspecto, se han presentado estas herramientas para Prosopis affinis Spreng.; Prosopis nigra Griseb y Vachellia caven Mol. Molina en bosques del Espinal de la provincia de Entre Ríos, Argentina (Sione et al., 2020 (1); Sione et al., 2020 (2)).

El almacenamiento neto de carbono orgánico en los bosques depende del manejo dado a la cobertura vegetal, edad, distribución de tamaños de individuos, estructura y composición de ésta (Cadena y Ángeles, 2005; Razo-Zárate et al., 2013). En el Espinal, los bosques nativos presentan un alto grado de heterogeneidad respecto a su densidad, área basal y clases diamétricas, lo cual puede atribuirse a las condiciones ecológicas sumadas a la historia de uso y manejo de cada sitio (Ledesma et al., 2007). En consecuencia, resulta de máximo interés valorar en forma precisa y confiable la capacidad de fijación de $\mathrm{C}$ en bosques con características diversas, para evaluar su potencial en el marco de desarrollo de proyectos de mitigación de cambio climático en distintas regiones. Para caracterizar de manera integral el rol de la vegetación boscosa en la dinámica del C, es importante determinar la tasa de captura de $\mathrm{CO}_{2}$, directamente relacionada con la velocidad de acumulación de biomasa. El ritmo con que se produce esta acumulación responde a numerosos factores, empezando por aquellos que rigen el desarrollo del proceso fotosintético (intensidad lumínica, temperatura, concentración de $\mathrm{CO}_{2}$ ) y de la respiración que hace uso de parte de los fotoasimilados, generando una producción primaria neta (Azcon Bieto y Talon, 2008). 
El balance positivo entre ambos procesos se evidencia en el crecimiento de los tejidos vegetales por unidad de tiempo, un parámetro medible que permite caracterizar la dinámica de los ecosistemas.

El Reporte Técnico de USDA (Janowiak et al., 2017) destaca la importancia de realizar mediciones seguras de la dinámica del C, por cuanto considera que, si bien los flujos de $\mathrm{C}$ son habitualmente muy pequeños en comparación con los depósitos, aún mínimos cambios en la magnitud de los flujos globales han provocado un profundo efecto sobre el ciclo global del C y han influido notablemente sobre el avance del cambio climático.

El objetivo de este estudio fue realizar una estimación del stock de $\mathrm{C}$ y la tasa anual de captura de $\mathrm{C}$ en distintos tipos de bosques del Espinal de Entre Ríos (Argentina).

\section{MATERIALES Y METODOS}

\section{Área de Estudio}

El trabajo se llevó a cabo en bosques nativos de la Provincia Fitogeográfica del Espinal, Distrito Ñandubay (Cabrera, 1976) localizado en la Provincia de Entre Ríos, Argentina. Se trata de una zona de clima templado, con una temperatura media diaria de $18,5^{\circ} \mathrm{C}$; y precipitaciones promedio anuales de $1100 \mathrm{~mm}$. La vegetación dominante es el bosque semixerofítico constituido principalmente por $P$. affinis "ñandubay" $y$ V. caven "espinillo", acompañado por $P$. nigra "algarrobo negro", Prosopis alba Griseb., y Celtis ehrenbergiana (Klotzsch) Liebm. En esta zona se verifica la presencia de suelos de los órdenes Vertisoles, Molisoles y Alfisoles (Plan Mapa de Suelos-Con- venio INTA Gobierno de Entre Ríos; 1990; 1998) lo cual, sumado a una profusa red hídrica le confiere una importante heterogeneidad ecológica

Se trabajó en seis bosques nativos caracterizados como bosques estables dado que presentan características correspondientes con un estado sucesional avanzado, de características similares a la vegetación prístina descripta para esta región fitogeográfica (Cabrera, 1976). A los fines de esta publicación serán denominados como Bosques 1; $2 ; 3 ; 4 ; 5$ y 6 .

\section{Caracterización de los bosques}

En cada uno de los seis bosques se efectuó un relevamiento de campo destinado a la determinación de la densidad total (DT) y la densidad relativa (DR) de las especies arbóreas. Se aplicó un método parcelario, aplicando una superficie de parcela de 1000 $\mathrm{m}^{2}$. En todos los individuos arbóreos presentes en la parcela se registró el diámetro basal a $15\left(\mathrm{~d}_{15}\right)$ y a $30\left(\mathrm{~d}_{30}\right) \mathrm{cm}$ desde la base del tronco, y el diámetro a la altura del pecho (DAP) a $130 \mathrm{~cm}$ desde la base. En los individuos de $P$. affinis y $P$. nigra con $\mathrm{d}_{30}$ superiores a $42 \mathrm{~cm}$, se registró también la altura de fuste, dado que así lo requieren los modelos alométricos utilizados para la estimación de la biomasa (Tabla 1).

En base al $\mathrm{d}_{15}$, se efectuó la clasificación de los individuos en cuatro Clases Diamétricas: I (menos de15 cm); II (15 a $25 \mathrm{~cm}$ ); III (25 a $35 \mathrm{~cm}$ ); y IV (más de $35 \mathrm{~cm}$ ); siguiendo la clasificación utilizada por Sione et al. (2011). 


\section{Cálculo de biomasa y stock de Carbono}

Para el cálculo de la biomasa arbórea se aplicaron modelos alométricos específicos para cada especie (Tabla 1). La biomasa se expresó en materia seca (MS).

Posteriormente, se calculó el contenido de $\mathrm{C}$ almacenado en la biomasa aérea de cada individuo. En el caso de $P$. affinis se tomó un valor fracción de carbono de 0,47 (Sione et al., 2019 (1)); mientras que para el resto de las especies se tomó el valor de 0,5 sugerido por el Intergovernmental $\mathrm{Pa}$ nel of Climatic Change (IPCC) en los lineamientos para la realización de inventarios de emisiones (1996). En base a los valores individuales de contenido de $\mathrm{C}$ y las densidades relativas de las especies presentes se obtuvo el stock total de C/ha para cada uno de los bosques evaluados.

\section{Cálculo de tasa de captura de C}

Se estimó la cantidad de $\mathrm{C}$ capturado por el estrato arbóreo de los seis bosques durante un período de un año, a partir del incremento anual de biomasa por individuo; calculado aplicando los modelos alométricos de estimación de biomasa sobre el nuevo valor de diámetro resultante del crecimiento experimentado por un individuo arbóreo en un año. Para $P$. nigra, $P$. affinis y $V$. caven se aplicaron los valores de incremento diamétrico anual por clase diamétrica determinados por Sione et al. (2011) en bosques del Espinal de Entre Ríos (Tabla 2).

Para Geoffroea decorticans (Gillies ex Hook. \& Arn.) Burkart se utilizó un valor de incremento diamétrico anual de $0,59 \mathrm{~cm}$ (Giraldo et al., 2006); y para A. quebracho blanco Schlecht, se aplicó el incremento

Tabla 1. Modelos alométricos de estimación de biomasa arbórea aérea individual por especie. Table 1. Allometric models for estimating individual aerial tree biomass for each species.

\begin{tabular}{|l|l|l|}
\hline \multicolumn{1}{|c|}{ Especie } & \multicolumn{1}{c|}{ Modelo } & \multicolumn{1}{c|}{ Autores } \\
\hline Prosopis affinis & Ln Biomasa $=-3,23+2,61^{*} \operatorname{Ln}\left(\mathrm{d}_{30}\right)$ & Sione et al., 2019 (1) \\
\hline Prosopis nigra & Ln Biomasa $=-2,86+2,60 * \operatorname{Ln}\left(\mathrm{d}_{30}\right)$ & Sione et al., 2020 (1) \\
\hline Vacchellia caven & Ln Biomasa $=-3,70+2,83^{*} \mathrm{Ln}\left(\mathrm{d}_{30}\right)$ & Sione et al., 2020 (2) \\
\hline $\begin{array}{l}\text { Aspidosperma quebracho } \\
\text { blanco }\end{array}$ & Biomasa $=0,0738697^{*} \mathrm{~d}_{15}{ }^{2,5939}$ & Gaillard de Benitez et al., 2000 \\
\hline Geoffroea decorticans & Ln Biomasa $=-3,11+2.55^{*} \ln \mathrm{d}_{15}$ & Iglesias y Barchuk, 2010 \\
\hline
\end{tabular}

Tabla 2. Incrementos diamétricos individuales por especie y clase diamétrica (Sione et al., 2011). Table 2: Individual diameter increases by species and diameter class (Sione et al., 2011).

\begin{tabular}{|c|c|c|c|}
\hline & \multicolumn{3}{|c|}{$\begin{array}{c}\text { Incremento diamétrico anual por } \\
\text { especie (cm/año) }\end{array}$} \\
\hline $\begin{array}{c}\text { Clase } \\
\text { Diamétrica }\end{array}$ & $\begin{array}{c}P . \\
\text { affinis }\end{array}$ & P. nigra & V. caven \\
\hline I & 0,1 & 0,45 & 0,145 \\
\hline II & 0,13 & 0,5 & 0,09 \\
\hline III & 0,09 & 0,3 & 0,05 \\
\hline IV & 0,075 & 0,4 & \\
\hline
\end{tabular}

Donde: Clase Diamétrica I: <15 cm; II (15 a 25 cm); III (25 a 35 cm); y IV (más de 35 cm) 
diamétrico anual sugerido por Araujo et al. (2007). Con la estimación del incremento individual anual de $\mathrm{C}$ almacenado, se obtuvo el valor de tasa anual de captura de C por hectárea para cada bosque y se hizo referencia a la cantidad del $\mathrm{CO}_{2}$ retenido por una hectárea.

\section{RESULTADOS}

\section{Caracterización estructural de los bosques}

La densidad total de los bosques en estudio varió entre 330 y 650 individuos/ha, registrándose en total 5 especies arbóreas que contribuyeron con distintas proporciones de DR (Tabla 3). El valor más alto de densidad relativa fue alcanzado por $P$. affinis en el bosque 1 (100\%). También presentó la mayor DR de otros tres bosques. Por su parte, $V$. caven aportó la mayor DR en el bosque 5; y P. nigra en el bosque 6 (Tabla 3).

La distribución de clases diamétricas en los bosques estudiados presentó una gran heterogeneidad. Las clases I, II y III fueron las más numerosas en general. Si bien no se registraron individuos de Clase I (menos de $15 \mathrm{~cm}$ de $\mathrm{d}_{15}$ ) en el Bosque 5, el 65\% de los individuos del Bosque 2 (Figura 1 b) pertenecieron a este rango. Por su parte, la clase V, presentó registros en todos los bosques, aunque en menos del $13 \%$ en todos los casos, excepto en el Bosque 3, donde representó un 36\% de la densidad total (Figura 1).

La clase IV presentó mayoría de individuos de $P$. nigra y $P$. affinis, siendo las especies que alcanzaron mayores valores de $\mathrm{d}_{15}$. El Bosque 1 por su parte, se presentó como un estrato arbóreo monoespecífico de P. affinis, en el cual un $75 \%$ de los árboles fue asignado a las clases I y II. En el Bosque 5 , donde $V$. caven presentó el valor más alto de densidad relativa (56,82\%), un 52\% de los individuos correspondió a la clase

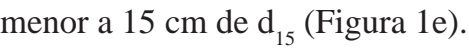

\section{Estimación de Biomasa y Stock de Carbono}

La biomasa arbórea aérea de los bosques estudiados osciló entre 52,13 y 111,82 t MS/ha (Tabla 4), arrojando un promedio de 92,68 \pm 23,13 t MS/ha. Esta variabilidad se atribuye a la heterogeneidad estructural de estos ecosistemas, dada por la densidad arbórea, la contribución de las especies a la densidad arbórea total y a su composición por clases diamétricas (Figura 1).

Tabla 3. Densidad Total (DT) y Densidad Relativa (DR) por especie en seis bosques nativos del Espinal (Entre Ríos, Argentina).

Table 3. Total tree density an relative tree density in six Espinal native forest (Entre Ríos, Argentina).

\begin{tabular}{|c|c|c|c|c|c|c|}
\hline & \multicolumn{6}{|c|}{ BOSQUES } \\
\hline & 1 & 2 & 3 & 4 & 5 & 6 \\
\hline DT (ind/ha) & 650 & 490 & 330 & 620 & 440 & 386 \\
\hline \multicolumn{7}{|c|}{ DR (\%) } \\
\hline P. nigra & 0 & 32,65 & 24,40 & 6,45 & 20,45 & 45,59 \\
\hline$P$. affinis & 100 & 65,31 & 66,51 & 82,26 & 22,72 & 24,61 \\
\hline V. caven & 0 & 2,04 & 9,09 & 11,29 & 56,83 & 24,61 \\
\hline G. decorticans & 0 & 0 & 0 & 0 & 0 & 4,67 \\
\hline A.quebracho blanco & 0 & 0 & 0 & 0 & 0 & 0,52 \\
\hline
\end{tabular}


Si bien la mayor DT se registró en el Bosque 1 (650 individuos/ha), el mayor valor de biomasa total por hectárea correspondió al Bosque 3, lo que puede atribuirse a la proporción de individuos de la Clase IV (más de $35 \mathrm{~cm}$ de $\mathrm{d}_{15}$ ), clase que contribuyó

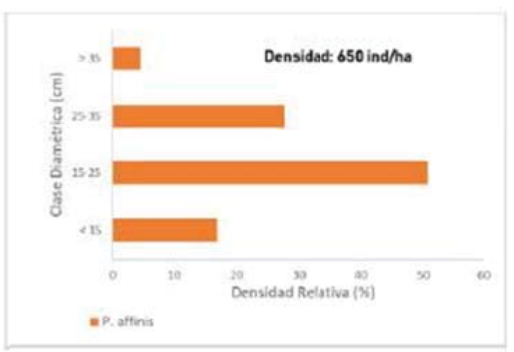

c.

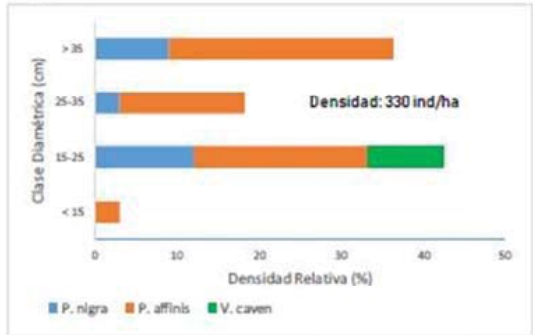

e.

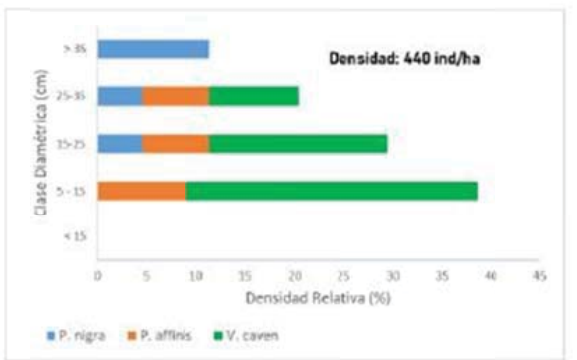

con el 72,85\% de la biomasa total. Asimismo, la alta proporción de individuos con $d_{15}$ menor a $25 \mathrm{~cm}$ en el Bosque 4, explica que su valor de biomasa total haya resultado el segundo más bajo a pesar de contar con una DT de 620 ind/ha.

b.

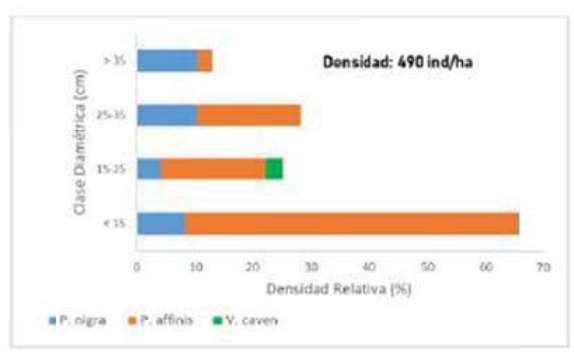

d.

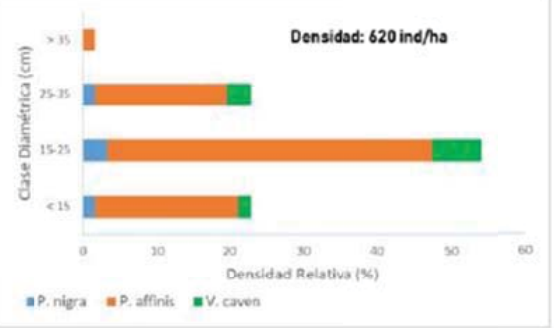

f.

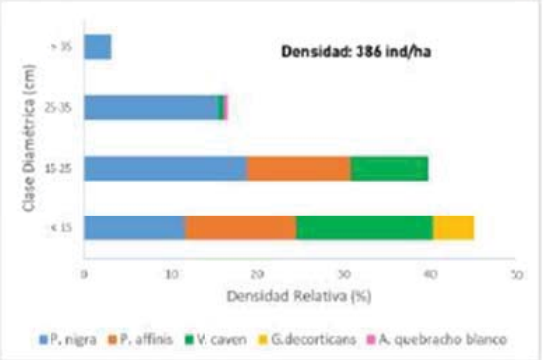

Figura 1. Distribución porcentual de clases diamétricas por especie en seis bosques del Espinal de Entre Ríos (Argentina). a: Bosque 1; b: Bosque 2; c: Bosque 3; d: Bosque 4; e: Bosque 5; f: Bosque 6.

Figure 1. Percentaje distribution of diameter classes by species in six Espinal native foret (Entre Ríoas, Argentine). a: Forest 1; b: Forest 2; c: Forest 3; d: Forest 4; d: Forest 5; e: Forest 6. 
S.G. Ledesma et al.

Tabla 4. Biomasa arbórea aérea total y contribución porcentual por especie en seis bosques nativos del Espinal (Entre Ríos, Argentina).

Table 4. Total tree aerial biomass and percentage contribution by species in six Espinal native forests (Entre Ríos, Argentina).

\begin{tabular}{|c|c|c|c|c|c|c|}
\hline & \multicolumn{7}{|c|}{ BOSQUES } \\
\hline & 1 & 2 & 3 & 4 & 5 & 6 \\
\hline $\begin{array}{c}\text { Biomasa arbórea aérea } \\
\text { (t MS/ha) }\end{array}$ & 108,26 & 102,81 & 111,82 & 78,05 & 102,98 & 52,13 \\
\hline \multicolumn{7}{|c|}{ Biomasa relativa por especie (\%) } \\
\hline P. nigra & 0 & 68,71 & 43,65 & 10,20 & 62,78 & 81,71 \\
\hline P. affinis & 100 & 30,55 & 52,70 & 79,16 & 13,33 & 2,92 \\
\hline V. caven & 0 & 0,74 & 3,65 & 10,64 & 23,89 & 13,32 \\
\hline G. decorticans & 0 & 0 & 0 & 0 & 0 & 1,16 \\
\hline A.quebracho blanco & 0 & 0 & 0 & 0 & 0 & 0,89 \\
\hline
\end{tabular}

La especie $P$. nigra aportó la mayor proporción de biomasa en tres de los bosques, aportando entre el 62,78 y 81,71\% del total. Por su parte, $P$. affinis, además de constituir el total de la biomasa presente en el Bosque 1, aportó más de la mitad de la biomasa total de los Bosques 3 y 4. Esto concuerda con la descripción de las DR, donde ambas especies presentaron la mayor proporción de individuos arbóreos. Excepto en el Bosque 1 que resultó una comunidad monoespecífica de $P$. affinis, la especie $V$. caven contribuyó a la biomasa total en todos los bosques, pero en ningún caso superó el $25 \%$, dada su bajo aporte de densidad en los bosques 2; 3; 4 y 6 . En el Bosque 5, aunque alcanzó el 56,82\% de la densidad arbórea total, el 84\% de esos individuos correspondieron a diámetros basales menores a 25 $\mathrm{cm}$. En general, la proporción de $P$. nigra resultó decisiva en el valor de biomasa no sólo porque fue la especie que presentó los valores diamétricos más altos, sino porque los modelos alométricos indican que, a valores similares de diámetro, esta especie alcanza mayor biomasa individual.

El stock de $\mathrm{C}$ estimado en los bosques varió entre 26,02 y 52,78 t C/ha (Tabla 5), arrojando un promedio de 43,99 $\pm 10,43$ t C/ ha. Este stock representa 161,44 t CO/ha capturados de la atmósfera para producir la actual biomasa.

\section{Tasa de Captura Anual de Carbono}

Las tasas de captura de $\mathrm{C}$ de los bosques variaron entre 0,53 y 0,95 t C/ha/año, con un promedio de 0,75 $\pm 0,17$ t C/ha/año. Esto indica que, en promedio, 1 ha de bosque nativo del Espinal entrerriano secuestra 2,75 t

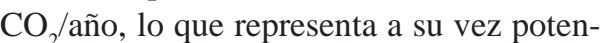
ciales emisiones de este GEI a la atmósfera en caso de deforestación.

Las tasas de captura de $\mathrm{C}$ resultaron menores en los bosques 1 y 4 (0,53 y 0,60 t C/ha/año), a pesar de sus mayores valores de densidad arbórea. Esto podría estar relacionado con la mayor contribución de la especie $P$. affinis en sus clases diamétricas I y II, que son las clases en las que se han determinado incrementos diamétricos relativamente bajos (Tabla 2). El valor más alto de captura anual de $\mathrm{C}$ correspondió al bosque 2, que presentó mayor proporción de individuos jóvenes, de la clase diamétrica I ( $\left.\mathrm{d}_{15}<15 \mathrm{~cm}\right)$. Los mayores valores de incrementos anuales de diámetro en la clase I en $P$. nigra y $V$. caven, explican un mayor ritmo de captura de $\mathrm{C}$ en el tejido vegetal.

| Revista FAVE - Ciencias Agrarias 20 (1) 2021 
Tabla 5. Stock y Tasa anual de captura de Carbono en seis bosques del Espinal (Entre Ríos, Argentina).

Table 5. Stock and annual C capture rate in six Espinal native forest of Espinal (Entre Ríos, Argentina).

\begin{tabular}{|l|c|c|c|c|c|c|}
\hline & \multicolumn{7}{|c|}{ BOSQUES } \\
\hline & 1 & 2 & 3 & 4 & 5 & 6 \\
\hline Stock de Carbono (t/ha) & 51,10 & 48,53 & 52,78 & 36,84 & 48,61 & 26,02 \\
\hline $\begin{array}{l}\text { Tasa de captura anual de C } \\
\text { (t/ha/año) }\end{array}$ & 0,53 & 0,95 & 0,71 & 0,60 & 0,81 & 0,92 \\
\hline
\end{tabular}

\section{DISCUSIÓN}

El presente trabajo aporta a la valoración precisa del servicio ecosistémico de los bosques nativos del Espinal como secuestradores y fijadores de $\mathrm{CO}_{2}$. Se observó la importancia de contar con las herramientas locales de estimación tales como los modelos alométricos específicos; valores de fracción de $\mathrm{C}$ y tasas de crecimiento que han sido determinadas para las especies arbóreas nativas desarrolladas en este tipo de ecosistemas. En los bosques analizados en este estudio, la biomasa arbórea aérea varió entre 52,13 y 111,82 t MS/ha. El valor promedio obtenido (92,68 t MS/ha) resulta similar al estimado por la Unidad de Manejo del Sistema de Evaluación Forestal UMSEF (2007) para el Parque Chaqueño (98,80 t MS/ha), atribuible a la similitud entre ambas regiones fitogeográficas. Para Argentina, dada su amplia variabilidad ecológica, se han estimado valores de biomasa aérea de las tierras forestales de las distintas ecorregiones que van desde 98,80 t MS/ha en el Parque Chaqueño, pasando por 172,87 y 266,24 t MS/ha para la Selva Tucumano Boliviana y la Selva Misionera respectivamente; y alcanzando las 540,20 t MS/ha en los bosques andino patagónicos (Secretaría de Ambiente y Desarrollo Sustentable de la
Nación, 2007). Estudios similares en otras regiones boscosas de América han informado sobre la gran variabilidad del stock de biomasa y $\mathrm{C}$ de las masas forestales, fruto de los diferentes tipos de bosques en relación a las diversas condiciones ecológicas del continente (Brown, 1997). Para los bosques tropicales de Brasil, Costa Rica y Guatemala, una revisión recopila contenidos de biomasa arbórea que varían entre 112 y 563 t MS/ha (Arreaga Gramajo, 2002). En sintonía con ello, han sido reportados estudios en bosques tropicales de Colombia donde los bosques primarios de montaña presentaron 319 t MS/ha de biomasa, y los secundarios alcanzaron las 130 t MS/ha (Yepes et al., 2010). Sin embargo, dentro de ese país, para agroecosistemas ganaderos de bosques de Acacia mangium se han estimado valores de biomasa arbórea de 70 t MS /ha (Giraldo et al., 2006), reflejando la influencia de la intervención antrópica y el tipo de especie arbórea dominante. Por otro lado, los bosques fríos de montaña de Perú (2650 a 3200 m s.n.m.) contienen hasta $146 \mathrm{t}$ MS/ha de biomasa, mientras que en Chile se reportan bosques de coihüe cuya biomasa arbórea alcanza hasta 673 t MS/ ha (Altamirano et al., 2015; Perez-Quezada et al., 2015). Estos datos refuerzan lo que previamente se había informado (Schlegel, 
2001), cuando un estudio estimó valores de 140 a 503 t MS/ha para este tipo de bosques siempreverdes de Chile.

Diversos estudios han estimado el contenido de $\mathrm{C}$ en los ecosistemas boscosos de las ecorregiones argentinas en pos de valorarlos como sumideros de C (Bonino, 2006; Gasparri y Manghi, 2004; Gasparri et al., 2008; Gasparri y Baldi, 2013; Manrique et al., 2011; Vicente et al., 2011). En el presente estudio, se estimó un almacenamiento promedio de 43,99 $\pm 10,43 \mathrm{t} \mathrm{C/}$ ha en bosques del Espinal entrerriano. Las estimaciones que integran el Informe PNUD (2017), determinaron stock de $C$ de 59,2 t/ha en el Parque Chaqueño, 182,9 t/ ha en Bosques Patagónicos y 139,3 t/ha en las Yungas (Gasparri et al., 2008). Por su parte las estimaciones realizadas para la ecorregión Chaqueña por Bonino (2006), determinaron valores de 30,31 t C/ha en los bosques primarios de esa región. En un rango similar se encuentran los reportes para los bosques del Chaco Seco (Conti et al., 2014). Estos autores calcularon un pool de, 22,13 t C/ha en el estrato arbóreo de bosques primarios y de $18,75 \mathrm{t} \mathrm{C} /$ ha en bosques secundarios de la región árida de la Provincia de Córdoba. En bosques del Chaco Salteño se han determinado valores similares (48 t C/ha) (Manrique et al., 2011).

Se ha detectado una carencia de información en relación a este tema para los bosques semixerofíticos del Espinal, tan es así que para la construcción del documento "Nivel de referencia de emisiones forestales de la República Argentina” presentado en 2019, la fuente de información sobre el nivel de reserva de C del Espinal (53,03 t C/ ha) surge de las determinaciones realizadas en 2006 durante el desarrollo del PIBN (Secretaría de Ambiente y Desarrollo de la Nación, 2007).
En relación a la captura anual de C, directamente relacionada con la participación del ecosistema boscoso en la dinámica de los GEI, es importante destacar la variabilidad que muestra la tasa anual de fijación de $C$ en la biomasa arbórea según el tipo de vegetación, localización geográfica, estado sucesional, condiciones de crecimiento y frecuencia y magnitud de disturbios (FONAM, 2005; Baral et al., 2009; Pompa-García y Sigala-Rodríguez, 2017; Torres-Torres et al., 2017). En el presente estudio, se estimó una captura anual promedio de $0,75 \pm 0,17$ t C/ha/año. Un estudio de carácter regional (Poorter et al., 2016) estimó valores de hasta 3,06 t C/ha/año capturados por bosques secundarios tropicales de América; mientras que otros autores han estimado una captura anual potencial de 1,54 t C/ha en México (Ordóñez Díaz et al., 2015) citando a la edad de la comunidad y a la localización geográfica como factores condicionantes principales. En los bosques de altura de México, en los que se han determinado altos niveles de acumulación de C, se han reportado valores de captura de 2 t C/ha/año, especialmente en comunidades arbóreas dominadas por Polylepis sp. (Fehse et al., 2002). Estos datos confirman también lo que había rescatado un estudio de bosques de regeneración en México; en cuanto a que la tasa de fijación de $\mathrm{C}$ por medio de procesos de fotosíntesis es más alta en rodales jóvenes que en rodales maduros (Razo-Zárate et al., 2013), aunque el almacenamiento total de $\mathrm{C}$ en el sistema es mayor en los bosques maduros. Investigadores del área de la bioenergía (Kraxner et al., 2007) explican esta condición al decir que el potencial de fijación de carbono de un bosque es limitado, debido a su saturación y permanencia: cuando los bosques llegan a la madurez, bajo determinadas condicio- 
nes climáticas y de suelo, se ha alcanzado el máximo potencial de almacenamiento de carbono (en el supuesto de que no hayan sufrido intervenciones naturales o antrópicas). En base a este comportamiento, se elaboró una propuesta de manejo de bosques de la provincia de Salta (Argentina) con aprovechamiento energético (Manrique et al., 2014); que recomienda mantener poblaciones en constante recuperación de biomasa para cumplir con aquel objetivo de manejo y, a la vez, favorecer la captura de GEI.

Dada la carencia de estimaciones locales, en el área de bosques xerófilos de la Provincia Chaqueña se ha utilizado un valor de captura de 0,5 t C/ha/año para cuantificar el aporte de estos ecosistemas en el secuestro de C (Gasparri y Manghi, 2004), según lo sugerido por el IPCC. En Argentina son escasos los estudios que abordan la cuantificación de la tasa anual de captura de C por parte de una comunidad arbórea nativa. Entre la escasa información disponible, se puede citar el estudio realizado en la selva Paranaense de Misiones (Argentina), donde Vaccaro et al. (2014) reportaron tasas de captura de $\mathrm{C}$ comprendidas entre 1 y 6 t C/ha/año, evidenciando la variabilidad que implica el tipo de vegetación y la etapa sucesional.

\section{CONCLUSIONES}

En este trabajo se determinó una importante heterogeneidad entre comunidades boscosas del Espinal en cuanto a la biomasa arbórea aérea y el C almacenado en la misma, acorde con la variabilidad observada en la DT y en la contribución de las especies a esa densidad. Esta variabilidad se reflejó en la biomasa arbórea aérea, que varió entre 52,13 y 111,82 t MS/ha. La contribución de la especie $P$. nigra y la estructura diamétrica de las poblaciones de esa especie resultaron determinantes para el valor final de biomasa y carbono almacenado en los bosques analizados.

En función de los valores del C almacenado en la biomasa arbórea de los bosques nativos del Espinal, mantener esta vegetación en pie y sin degradación, implica evitar emisiones de entre 95,49 y 193,70 t de $\mathrm{CO}_{2}$ /ha hacia la atmósfera. Asimismo, a partir de los valores de fijación anual de C en la biomasa, se estima que una hectárea de bosque nativo está en condiciones de secuestrar y retener en sus tejidos vegetales entre 1,94 y 3,49 t de $\mathrm{CO}_{2} /$ ha cada año.

Este trabajo comprobó la importancia de la generación e integración de información y herramientas de carácter local para la determinación precisa de la biomasa arbórea aérea de distintos tipos de bosques del Espinal entrerriano y su capacidad de captura de C. Los modelos alométricos de estimación de biomasa aérea y los valores de fracción de $\mathrm{C}$ utilizados resultaron adecuados para valorar el servicio ecosistémico de los bosques del Espinal como secuestradores y fijadores de $\mathrm{CO}_{2}$. 
La información obtenida resulta valiosa para ser utilizada por quienes deben diseñar estrategias de manejo de bosques nativos con vistas a la mitigación del cambio climático global.

\section{AGRADECIMIENTOS}

Este estudio se llevó a cabo en el marco del PID UNER 2223 "Captura y fijación de carbono como servicio ambiental de los bosques nativos del Espinal" financiado por la Universidad Nacional de Entre Ríos.

\section{BIBLIOGRAFÍA}

Altamirano, A.; Schlegel, B.; Thiers, Ó.; Miranda, A.; Pilquinao, B.; Orrego, R.; Rocha, C. 2015. Disponibilidad y potencial energético de la biomasa del bosque nativo para el desarrollo de la dendroenergía en el centro-sur de Chile. Bosque (Valdivia), 36(2), 223-237.

Araujo, P.; M. J. de Galíndez Iturre, M. 2007. Crecimiento de las especies principales de un bosque en regeneración del Chaco Santiagueño. Quebracho N¹4 36-46

Arreaga Gramajo, W.E. 2002. Almacenamiento de carbono em bosques com manejo florestal sostenible em a Reserva de Biosfera Maya, Petén, Guatemala. [Tesis Mag. Sc.] [Costa Rica]:CATIE.

Azcon Bieto, J. Talon, M. 2008: Fundamentos de Fisiología Vegetal. 2da.Edición Madrid: Interamericana-McGraw-Hill. 651 pp.

Barala, A.;Guha, G.. 2004. Trees for carbon sequestration or fossil fuel substitution: the issue of cost vs. carbon benefit. Biomass and Bioenergy $2741-55$
Baral, S.; Malla, R.; Ranabhat, S. 2009. Above-ground carbon stock assessment in different forest types of Nepal. Banko Janakari, 19(2), 10-14.

Bishop, J.,Landell-Mills, N. 2003. Los servicios ambientales de los bosques: información general. En: S. Pagiola, J. Bishop, y N. Landell-Mills, editores. La Venta de Servicios Ambientales Forestales: Mecanismos Basados en el Mercado para la Conservación y el Desarrollo.[México]: INE-SEMARNAT. Pp: 43-75.

Bonino, E.E., 2006. Changes in carbon pools associated with a land-use gradient in the dry Chaco, Argentina. For. Ecol. Manage. 223, 183-189.

Brown, S.1997. Estimating biomass and biomass change of tropical forests. Forest Resources Assessment Publication. Forestry Papers 134. FAO 55 pp.

Cabrera, A.L. 1976. Regiones fitogeográficas argentinas. En: Kugler WF Editores.Enciclopedia argentina de agricultura y jardinería. Tomo 2. 2a edición.. Buenos AiresAcme Fascículo 1. 1-85.

Cadena M., O.I.,Ángeles, G.. 2005. Almacenes de carbono en hojarasca en bosques manejados de Pinus patula en Zacualtipán, Hidalgo. Trabajo Presentado en: VII Congreso Mexicano de Recursos Forestales. México

Canadell, J.G.,Raupach, M.R.. 2008. Managing forests for climate change mitigation. Science 13;320(5882):1456-7

Conti, G.; Pérez-Harguindeguy, N.; Quètier, F.; Gorné, L.D.; Jaureguiberry, P. 2014. Large changes in carbon storage under different land-use regimes in subtropical seasonally dry forests of southern South America. Agriculture, ecosystems \& environment 197, 68-7 
Casiano-Domínguez, M., Paz-Pellat, F., RojoMartínez, M., Covaleda-Ocon, S., Aryal, D. 2018. El carbono de la biomasa aérea medido en cronosecuencias: primera estimación en México. Madera y Bosques, 24.

Fehse, J.; Hofstede, R. Aguirre, N.; Paladines, C.; Kooijman, A., Sevink, J. 2002. High altitude tropical secondary forests: a competitive carbon sink. Forest Ecology and Management 163(1):9-25

[FONAM]Fondo Nacional del Ambiente. 2005. Boletín CO2 comercio. Dedicado a Informar Sobre las Oportunidades del Mercado de Carbono. [Consulta 20 de diciembre de 2019]. http://www.fonamperu.org/ general/ mdl/documentos/guia\%20 MDL.pd.

Gaillard de Benitez, C., Pece, M., Juárez de Galíndez, M., Maldonado, A., Acosta, V.H., Gómez, A., 2002. Biomasa aérea de ejemplares de quebracho blanco (Aspidosperma quebracho-blanco) en dos localidades del parque chaqueño seco. Quebracho 9, 115-127

Gasparri, I., Manghi, E. 2004. Estimación de volumen, biomasa y contenido de carbono de las regiones forestales argentinas. Argentina: Dirección de Bosques Secretaría de Ambiente y Desarrollo Sustentable. Unidad de Manejo del Sistema de Evaluación Forestal.

Gasparri, N., Grau, H., Manghi, E. 2008. Carbon pools and emissions from deforestation in extra-tropical forests of northern Argentina between 1900 and 2005. Ecosystems 11, 1247-1261.

Gasparri, N., Baldi, G. 2013. Regional patterns and controls of biomass in semiarid woodlands: lessons from the Northern Argentina Dry Chaco. Reg Environ Change 13:1131-1144.

Giraldo, L.A.; Zapata, M.; Montoya, E. 2006. Estimación de la captura y flujo de carbono en silvopastoreo de Acacia mangium asociada con Brachiaria dyctioneura en Colombia. Pastos y Forrajes, vol. 29, núm. 4. 421-435
Ibárcena Escudero, M.Scheelje Bravo, J.. El cambio climático principales causantes, consecuencias y compromisos de los países involucrados. [Consultado el 09/01/2020). http://www.fao.org/3/XII/0523-B2.htm

Iglesias, M.R., Barchuk, A.H., 2010. Estimación de la biomasa aérea de seis leguminosas leñosas del Chaco Árido (Argentina). Ecol. Austr. 20, 71-79.

[IPCC] Intergovernmental Panel on Climate Change. 1996. Guidelines for National Greenhouse Gas Inventories. [Consultado el 09/01/2020] https://www.ipcc-nggip.iges. or.jp/public/gl/invs4.html

Janowiak, .; Connelly, W.; Dante-Wood, K.; Domke, G.; Giardina, C.; Kayler, Z.; Marcinkowski, K.; Ontl, T.; Rodriguez-Franco, C.; Swanston, C.; Woodall, C., Buford, M. 2017. Considering Forest and Grassland Carbon in Land Management. U.S. Department of Agriculture Forest Service General Technical Report WO-95. 69pp

Kraxner, F.; Nilsson, S. y M. Obersteiner. 2003. Negative emissions from BioEnergy use, carbon capture and sequestration (BECS) - the case of biomass production by sustainable forest management from semi-natural temperate forests. Biomass and Bioenergy Volume 24 Issues 4-5 285-296.

Ledesma, S.G., Muracciole, B., Dorsch, A.F., Sabattini, R., Cottani, F., Sione, S.M., Fortini, C. 2007. Caracterización estructural de montes nativos de Entre Ríos. Revista Científica Agropecuaria, 11 (2), 129-139

Loghersio, G. Cambio climático: El rol de los bosques como sumideros de carbono. [Consultado el 01/02/2020]. http://www.ciefap. org.ar/index.php/articulo-91.

Manrique, S., Franco, J., Núñez, V., Seghezzo, L., 2011. Potential of native forests for the mitigation of greenhouse gases in Salta, Argentina. Biomass Bioenerg. 35, 2184-2193. 
Manrique, S.; Franco, J.; Nuñez, V. 2014. Biomass feedstock availability for the supply of bioenergy in Lerma Valley (Salta, Argentina): an evaluation for energy territorial planning”. American Journal of Biomass and Bioenergy Vol. $334-57$

Martin, A. R. y S.C. Thomas. 2011. A Reassessment of Carbon Content in Tropical Trees. PLoS ONE 6(8): e23533. https://doi. org/10.1371/journal.pone.0023533

Moroni, M.T. 2013. Simple models of the role of forests and wood products in greenhouse gas mitigation. Australian Forestry, 76:1, 50-57

Ordóñez Díaz, J.; Rivera Vázquez, R.; Tapia Medina M. y L. Ahedo Hernández. 2015. Contenido y captura potencial de carbono en la biomasa forestal de San Pedro Jacuaro, Michoacán. Rev. Mex. de Cienc. Forestales vol.6 no.32. 7-16.

Perez-Quezada J., Olguín, S.; Fuentes J. P.; Galleguillos M. 2015. Tree carbon stock in evergreen forests of Chiloé, Chile. Bosque 36(1):27-39.

Plan Mapa de Suelos-Convenio INTA Gobierno de Entre Ríos. 1990. Carta de Suelos de la República Argentina, Departamento La Paz, Provincia de Entre Ríos. Tomos I y II. EEA Paraná INTA. Serie Relevamiento de Recursos Naturales $N^{\circ}$ 7, 321 pp

Plan Mapa de Suelos-Convenio INTA Gobierno de Entre Ríos. 1998. Carta de Suelos de la República Argentina, Departamento Paraná, Provincia de Entre Ríos. Acuerdo Complementario del Convenio INTA - Gobierno de Entre Ríos, EEA Paraná, Serie Relevamiento de Recursos Naturales No 17, 114 pp

PNUD Argentina. Los Bosques Nativos de Argentina en el marco del proceso de Reducción de Emisiones derivadas de la Deforestación y la Degradación [Consultado el 10/01/2020]. http://www.undp.org/content/dam/argentina/Publications/Energia\%20y\%20Desarrollo\%20Sostenible/brief-08-cambios.pdf
Pompa-García, M.; Sigala-Rodríguez, J.A. 2017. Variation of carbon uptake from forest species in Mexico: a review. Madera y Bosques 23-2. pp 225-235

Poorter, L.; Bongers, F.; Aide, M., Almeyda Zambrano, a.; Balvanera, P.; Becknell, J.; Boukili, V.; Brancalion, P.; Broadbent, E.; Chazdon, R. et al. 2016. Biomass resilience of Neotropical secondary forests. [Consultado el 06/01/2020]. Nature. doi:10.1038/ nature 16512

Razo-Zárate; R.; Gordillo-Martinez, A.; Rodriguez-Laguna, R.; Maycotte-Morales, C.; Acevedo-Sandoval, O.. 2013. Estimación de biomasa y carbono almacenado en árboles de oyamel afectados por el fuego en el Parque Nacional "El Chico”, Hidalgo, México. Madera y Bosques 19(2):73-86 73

Risio Allione, L. 2002. Cuantificación de biomasa y carbono en bosques nativos de Prosopis caldenia (Burkart) en la Pampa semiárida, Argentina. [Tesis Dr] en Instituto [Valladolid]: Universitario de Investigación Gestión Forestal Sostenible Universidad de Valladolid. $74 \mathrm{p}$.

Sabattini, R.; Sione, S.; Ledesma, S.; Sabattini, J.; Wilson, M. 2016. Estimación de la pérdida de superficie de bosques nativos y tasa de deforestación en la cuenca del Arroyo Estacas (Entre Ríos, Argentina). Revista Científica Agropecuaria 20(1-2): 45-56.

Schlegel, B. 2001. Estimación de la biomasa y carbono en bosques del tipo forestal siempreverde. Trabajo presentado en: Simposio Internacional Medición y Monitoreo de la Captura de Carbono en Ecosistemas Forestales. Valdivia, Chile.

Secretaría de Ambiente y Desarrollo Sustentable de la Nación. 2007. Primer Inventario nacional de bosques nativos. Inventario de campo de la Región del Espinal Distritos Caldén y Ñandubay. Informe Regional Espinal Segunda Etapa. 1ra. Ed. [Buenos Aires] 
Segura Madrigal, M.; Andrade Castañeda, H. y C. Mojica Sánchez. 2019. Estructura, composición florística y almacenamiento de carbono en bosques nativos del páramo de Anaime, Tolima, Colombia. Ci. Fl. v. 29, n. 1, p. $157-168$

Sione, S.; Sabattini, R.; Ledesma, S.; Dorsch, A. 2011. Incremento anual de área basal de Prosopis nigra Griseb.; Prosopis affinis Sprengel y Acacia caven (Mol.) Molina, en función de la densidad arbórea y la estructura de clases diamétricas, en bosques nativos de Entre Ríos. YVYRARETA 18 1-8

Sione, S.; Andrade-Castañeda, H.; Ledesma, S.: Rosenberger, L.; Oszust, J.; M. Wilson, M. 2019 (1). Aerial biomass allometric models for Prosopis affinis Spreng.in native Espinal forests of Argentina. Revista Brasileira de Engenharia Agrícola e Ambiental v.23, n.6, p.467-473 DOI: http:// dx.doi.org/10.1590/1807-1929/agriambi. v23n6p467

Sione, S., Ledesma, S. G., Rosenberger, L. J., Oszust, J. D., Carpp, I. A., Wilson, M. G., Sasal, M. C. 2019 (2). Fracción de carbono en la biomasa de Prosopis affinis Sprengel (Fabaceae) en un bosque nativo del espinal (Argentina). Agronomía \& Ambiente, 39 (1), 6-15.

Sione, S.M.; Ledesma, S.G., Rosenberger, L.J.; Oszust, J.D.; Andrade, H.J.; Maciel, G.O.; Wilson, M.G. y M.C. Sasal. 2020 (1). Ecuaciones alométricas de biomasa aérea para Prosopis nigra Hieron. "algarrobo negro" (Fabaceae) en bosques de Entre Ríos (Argentina). Agronomía \& Ambiente 40 (1): 63-76.

Sione, S.M.; Ledesma, S.G., Rosenberger, L.J.; Oszust, J.D.; Andrade, H.J.; Maciel, G.O.; Wilson, M.G. 2020 (2). Modelos alométricos de biomasa para Vachellia caven Mol. Molina en bosques nativos del Espinal (Argentina). Quebracho 28 (1,2), 20-33.
Spracklen, D.V. y R. Righelato. 2014. Tropical montane forests are a larger than expected global carbon store. Biogeosciences 11 2741-2754.

Torres-Torres, J.; Mena-Mosquera, V. y E. Álvarez-Dávila. 2017. Carbono aéreo almacenado en tres bosques del Jardín Botánico del Pacífico, Chocó, Colombia. En: Entramado 13-1200-209 http://dx.doi.org/10.18041/entramado.2017v13n1.25110

Unidad de Manejo del Sistema de Evaluación Forestal UMSEF. Monitoreo de la superficie de bosque nativo de Argentina. Dirección de Bosques. Secretaría de Ambiente y Desarrollo Sustentable. [Consultado el 10/01/2020] https://www.argentina.gob.ar/ambiente/bosques/umsef

Vaccaro, S.; Arturi, M.; Goya, J.; Frangi, J.; Piccolo, G. 2014. Almacenaje de carbono en estadíos de la sucesión secundaria en la provincia de Misiones, Argentina. Interciencia Vol. 28 Nro. 9 521-527.

Vicente, G.; Engler, P.; Jaubertie, C. 2011. Valorización económica del Carbono secuestrado en el Distrito Ñandubay, Entre Ríos, como herramienta para un ordenamiento territorial. En: Laterra, E.; Jobbágy, G.; Paruelo, J. Editores Valoración de servicios ecosistémicos: conceptos, herramientas y aplicaciones para el ordenamiento territorial. Buenos Aires: INTA págs.581-60

Yepes, A. P., del Valle, J. I., Jaramillo, S. L., Orrego, S. A. 2010. Recuperación estructural en bosques sucesionales andinos de Porce (Antioquia, Colombia). Revista de Biología Tropical Vol. 58 Nro. 1 427-445 